\title{
On the class number of some real abelian number fields of prime conductors
}

\author{
by \\ StanislaV JakubeC (Bratislava)
}

1. Introduction. The aim of this paper is to prove two theorems on the class number $h_{K}$.

Theorem 1. Let $p=4 l+1$ and $l$ be odd primes. Let $K \subset \mathbb{Q}\left(\zeta_{p}+\zeta_{p}^{-1}\right)$, $[K: \mathbb{Q}]=l$ and let $h_{K}$ be the class number of the field $K$. Let $q$ be an odd prime with $3<q<\sqrt{p}$. If $q$ is a primitive root modulo $l$ then $q$ does not divide $h_{K}$.

Theorem 2. Let $p=6 l+1$ and $l$ be odd primes. Let $K \subset \mathbb{Q}\left(\zeta_{p}+\zeta_{p}^{-1}\right)$, $[K: \mathbb{Q}]=l$ and let $h_{K}$ be the class number of the field $K$. Let $q$ be an odd prime with $3<q<\sqrt{p} / 2$. If $q$ is a primitive root modulo $l$ then $q$ does not divide $h_{K}$.

Using Schinzel's conjecture for linear polynomials (see [5] and [4, p. 56]) we prove that for each prime $q$ there exist infinitely many prime numbers $p$ satisfying the assumptions of Theorems 1 and 2 .

Proposition. Assume that Schinzel's conjecture for linear polynomials holds true. Then, for any given prime $q>3$, there are infinitely many pairs of primes $(l, p)$ of the form $p=4 l+1$ (respectively, of the form $p=6 l+1$ ), for which $q$ is a primitive root modulo $l$.

Proof. Let $l=2 r+1$ where $r$ is an odd prime. Then $q$ is a primitive root modulo $l$ if and only if $q \not \equiv 0, \pm 1(\bmod l)$ and the Legendre symbol $\left(\frac{q}{l}\right)$ equals -1 .

Because $l \equiv 3(\bmod 4)$, by the quadratic reciprocity law we have

$$
\left(\frac{q}{l}\right)=\left(\frac{-1}{q}\right)\left(\frac{l}{q}\right)
$$

2010 Mathematics Subject Classification: Primary 11R29.

Key words and phrases: class number. 
Let the residues modulo $q$ be represented by odd numbers $\{1,3, \ldots$, $2 q-1\}$. Let $z \in\{1,3, \ldots, 2 q-1\}, z \neq q, z \neq 1, z \neq(q-1) / 4$. Put $r=f_{1}(X)=q X+\frac{z-1}{2}, l=f_{2}(X)=2 q X+z, p=f_{3}(X)=8 q X+4 z+1$, where $\left(\frac{-1}{q}\right)\left(\frac{z}{q}\right)=-1$.

If $z \neq 1, z \neq q, z \neq(q-1) / 4$ then the linear polynomials $f_{1}(X), f_{2}(X)$, $f_{3}(X)$ satisfy the assumptions of Schinzel's conjecture and consequently the prime numbers $q, l, p$ satisfy the assumptions of Theorem 1 . In the case of Theorem 2 we consider the polynomials $r=f_{1}(X)=q X+\frac{z-1}{2}, l=f_{2}(X)=2 q X+z, p=f_{3}(X)=12 q X+6 z+1$.

Our approach is based on the results [1] and [2] (see also [3]). Let $q$ be an odd prime. Let $j \mapsto A(j)$ be the $q$-periodic function defined by

$$
A(0)=0, \quad A(j)=\sum_{i=1}^{j} \frac{1}{i} \quad \text { for } j=1, \ldots, q-1 .
$$

Let $s$ be a rational $q$-integer. Put $A(s)=A(j)$ where $j$ is an integer, $0 \leq$ $j<q$, and $s \equiv j(\bmod q)$.

For $i=1, \ldots, q-1$ we have the congruence $A(i-1) \equiv A(q-i)(\bmod q)$. This implies that

$$
A\left(\frac{-i}{p}\right) \equiv A\left(\frac{-(p-i)}{p}\right)(\bmod q) \quad \text { for } i=1, \ldots, p-1 .
$$

From [1]-[3], we have

Proposition 1. Let $l, p, q$ be primes, $p \equiv 1(\bmod l), q \neq 2, q \neq l$, $q<p$. Suppose that $q$ is a primitive root modulo $l$. If $q$ divides $h_{K}$, and $[K: \mathbb{Q}]=l$, then

$$
\sum_{j \in X} A\left(\frac{-j}{p}\right) \equiv \sum_{j \in Y} A\left(\frac{-j}{p}\right)(\bmod q)
$$

for any cosets $X, Y \subset\{1, \ldots, p-1\}$ of the subgroup $H$ of index $l$ in $(\mathbb{Z} / p \mathbb{Z})^{*}$.

Proof of Theorem 1. Let $H=\{1,-1, a / b,-a / b\}$ be the subgroup of order four of $(\mathbb{Z} / p \mathbb{Z})^{*}$ where $p=a^{2}+b^{2}, a, b>0$. Then $b H=\{a, p-a$, $b, p-b\}$ and $x b H=\{a x, p-a x, b, p-b x\}$. By Proposition 1 and since $A(-i / p) \equiv A(-(p-i) / p)(\bmod q)$, the following congruence holds if $q \mid h_{K}$, for $x=1, \ldots,[\sqrt{p}]$ :

$$
A\left(\frac{-a}{p}\right)+A\left(\frac{-b}{p}\right) \equiv A\left(\frac{-a x}{p}\right)+A\left(\frac{-b x}{p}\right)(\bmod q) .
$$

Further let $B_{n}$ and $B_{n}(X)$ denote the Bernoulli numbers and Bernoulli polynomials (see [4]). 
Let $-a / p \equiv k(\bmod q)$ for an integer $k, 0 \leq k<q$, hence $A(-a / p) \equiv$ $A(k)(\bmod q)$, so

$$
A\left(\frac{-a}{p}\right) \equiv \sum_{i=1}^{k} i^{q-2} \equiv \frac{1}{q-1}\left(B_{q-1}(k+1)-B_{q-1}\right)(\bmod q) .
$$

Since $B_{n}(1-x)=(-1)^{n} B_{n}(x)$ we have

$$
\begin{aligned}
A\left(\frac{-a}{p}\right) & \equiv \frac{1}{q-1}\left(B_{q-1}\left(\frac{-a}{p}+1\right)-B_{q-1}\right) \\
& \equiv \frac{1}{q-1}\left(B_{q-1}\left(\frac{a}{p}\right)-B_{q-1}\right)(\bmod q) .
\end{aligned}
$$

Let $F(x)$ be the polynomial

$$
F(x)=B_{q-1}\left(\frac{a x}{p}\right)+B_{q-1}\left(\frac{b x}{p}\right)-B_{q-1}\left(\frac{a}{p}\right)-B_{q-1}\left(\frac{b}{p}\right) .
$$

The numbers $x=1, \ldots,[\sqrt{p}]$ are roots of $F(x)$ modulo $q$. As $\operatorname{deg} F(x)<q$ we see that $F(x)$ has more roots modulo $q$ than its degree. However, we will prove that $F(x)$ is not identically zero modulo $q$. The coefficient of $x^{q-3}$ in $F(x)$ is equal to

$$
c_{q-3}=\left(\begin{array}{c}
q-1 \\
2
\end{array}\right) B_{2} \frac{1}{p^{q-3}}\left(a^{q-3}+b^{q-3}\right) .
$$

We will prove that $c_{q-3} \not \equiv 0(\bmod q)$. This is so if $a b \equiv 0(\bmod q)$, since $a^{2}+b^{2}=p \not \equiv 0(\bmod q)$. If $a b \not \equiv 0(\bmod q)$, then

$$
a^{2} b^{2}\left(a^{q-3}+b^{q-3}\right) \equiv a^{2}+b^{2} \equiv p \not \equiv 0(\bmod q),
$$

hence $c_{q-3} \not \equiv 0(\bmod q)$.

Proof of Theorem 2. Let $H$ be the subgroup of $(\mathbb{Z} / p \mathbb{Z})^{*}$ of order six, $4 p=a^{2}+3 b^{2}, a, b>0$, hence $a^{2} / b^{2} \equiv-3(\bmod p)$. It follows that

$$
\frac{1}{2}\left(-1+\frac{a}{b}\right), \frac{1}{2}\left(-1-\frac{a}{b}\right) \in H \text {. }
$$

This implies that

$$
\left\{b, \frac{-b+a}{2}, \frac{a+b}{2}\right\} \subset b H \quad \text { and } \quad\left\{b, \frac{b-a}{2}, \frac{a+b}{2}\right\} \subset b H .
$$

Let us consider the case when all three numbers are positive, for example in the first triple. Since $a^{2}+3 b^{2}=4 p$, we have $a<2 \sqrt{p}, b<2 \sqrt{p},(-b+a) / 2$ $<2 \sqrt{p},(b+a) / 2<2 \sqrt{p}$. Just as in the proof of Theorem 1 , if $q \mid h_{K}$, then 
the polynomial

$$
\begin{aligned}
F(x)= & B_{q-1}\left(\frac{b x}{p}\right)+B_{q-1}\left(\frac{\frac{-b+a}{2} x}{p}\right)+B_{q-1}\left(\frac{\frac{b+a}{2} x}{p}\right) \\
& -B_{q-1}\left(\frac{b}{p}\right)-B_{q-1}\left(\frac{\frac{-b+a}{2}}{p}\right)-B_{q-1}\left(\frac{\frac{b+a}{2}}{p}\right)
\end{aligned}
$$

has modulo $q$ the roots $x=1, \ldots,[\sqrt{p} / 2]$. However, we will prove that $F(x)$ is not identically zero modulo $q$.

The coefficient of $x^{q-3}$ in $F(x)$ is equal to

$$
c_{q-3}=\left(\begin{array}{c}
q-1 \\
2
\end{array}\right) B_{2} \frac{1}{p^{q-3}}\left(b^{q-3}+\left(\frac{a-b}{2}\right)^{q-3}+\left(\frac{a+b}{2}\right)^{q-3}\right) .
$$

We will prove that $c_{q-3} \not \equiv 0(\bmod q)$. This is so if $b \frac{a-b}{2} \frac{a+b}{2} \equiv 0(\bmod q)$, since $a^{2}+3 b^{2}=4 p \not \equiv 0(\bmod q)$. If $b \frac{a-b}{2} \frac{a+b}{2} \not \equiv 0(\bmod q)$, then

$$
\begin{aligned}
& b^{2}(a-b)^{2}(a+b)^{2}\left(b^{q-3}+\left(\frac{a-b}{2}\right)^{q-3}+\left(\frac{a+b}{2}\right)^{q-3}\right) \\
& \equiv(a-b)^{2}(a+b)^{2}+4 b^{2}(a-b)^{2}+4 b^{2}(a+b)^{2} \equiv\left(a^{2}+3 b^{2}\right)^{2} \equiv 16 p^{2} \not \equiv(\bmod q),
\end{aligned}
$$
hence $c_{q-3} \not \equiv 0(\bmod q)$.

Acknowledgments. The author thanks the referees for their remarks that improved the readability of the paper.

\section{References}

[1] S. Jakubec, On divisibility of class number of real abelian fields of prime conductor, Abh. Math. Sem. Univ. Hamburg 63 (1993), 67-86.

[2] -, On divisibility of the class number $h^{+}$of the real cyclotomic fields of prime degree l, Math. Comp. 67 (1998), 369-398.

[3] T. Metsänkylä, An application of the p-adic class number formula, Manuscripta Math. 93 (1997), 481-498.

[4] P. Ribenboim, 13 Lectures on Fermat's Last Theorem, Springer, New York, 1979.

[5] A. Schinzel et W. Sierpiński, Sur certaines hypothèses concernant les nombres premiers, Acta Arith. 4 (1958), 185-208; Corrigendum, ibid. 5 (1960), 259.

Stanislav Jakubec

Mathematical Institute

Slovak Academy of Sciences

Štefánikova 49

81473 Bratislava, Slovakia

E-mail: jakubec@mat.savba.sk 